DOI: $10.18027 / 2224-5057-2021-11-3 s 2-24$

Цитирование: Гордеев С.С., Бесова Н.С., Глебовская В.В., Горбунова В.А.,, Мамедли 3.3., Ткачев С.И. и соавт. Практические рекомендации по лечению рака анального канала и кожи перианальной области. Злокачественные опухоли : Практические рекомендации RUSSCO \#3s2, 2021 (том 11). 24

\title{
ПРАКТИЧЕСКИЕ РЕКОМЕНДАЦИИ ПО ЛЕЧЕНИЮ РАКА АНАЛЬНОГО КАНАЛА И КОЖИ ПЕРИАНАЛЬНОЙ ОБЛАСТИ
}

Коллектив авторов: Гордеев С.С., Бесова Н.С., Глебовская В.В., Горбунова В.А.,, Мамедли 3.3., Ткачев С.И., Федянин М.Ю., Черных М.В.

Ключевые слова: плоскоклеточный рак, анальный канал, кожа перианальной области, химиотерапия, химиолучевая терапия

\section{1. КЛАССИФИКАЦИЯ И СТАДИРОВАНИЕ}

Стадирование рака анального канала и перианальной кожи должно проводиться по системе TNM (8 редакция, 2017 г.) (табл. 1). Стадирование по параметрам Т и N (табл. 2) осуществляется на основании данных МРТ малого таза. Рак перианальной кожи определяется как опухоль, располагающаяся в пределах 5 см от анодермальной линии.

Таблица 1. Характеристика параметров системы TNM для рака анального канала и перианальной кожи

\begin{tabular}{|c|c|c|}
\hline & 7-я редакция TNM & 8-я редакция TNM \\
\hline \multicolumn{3}{|c|}{ Параметр Т-характеристика первичной опухоли } \\
\hline Tx & Невозможно оценить первичную опухоль & Невозможно оценить первичную опухоль \\
\hline TO & Первичная опухоль не определяется & \\
\hline Tis & Рак in situ & $\begin{array}{l}\text { Рак in situ, болезнь Боуэна или анальная } \\
\text { интраэпителиальная неоплазия тяжёлой } \\
\text { степени (HSIL/AIN 2-3) }\end{array}$ \\
\hline T1 & Опухоль $\leq 2$ см в наибольшем измерении & Опухоль $\leq 2$ см в наибольшем измерении \\
\hline $\mathrm{T} 2$ & $\begin{array}{l}\text { Опухоль }>2 \text { см, но } \leq 5 \text { см в наибольшем } \\
\text { измерении }\end{array}$ & $\begin{array}{l}\text { Опухоль }>2 \text { см, но } \leq 5 \text { см в наибольшем } \\
\text { измерении }\end{array}$ \\
\hline T3 & Опухоль > 5 см в наибольшем измерении & Опухоль > 5 см в наибольшем измерении \\
\hline T4 & $\begin{array}{l}\text { Опухоль любого размера с инвазией в соседние } \\
\text { органы (влагалище, уретра, мочевой пузырь и т. д.) }\end{array}$ & $\begin{array}{l}\text { Опухоль любого размера с инвазией в соседние } \\
\text { органы (влагалище, уретра, мочевой пузырь и т. д.) }\end{array}$ \\
\hline
\end{tabular}




\begin{tabular}{|c|c|c|}
\hline & 7-я редакция TNM & 8-я редакция TNM \\
\hline \multicolumn{3}{|c|}{$\begin{array}{l}\text { Параметр N-характеристика регионарных лимфоузлов: лимфоузлы мезоректума (аноректальные, } \\
\text { сакральные), внутренние и наружные подвздошные (гипогастральные), паховые. }\end{array}$} \\
\hline $\mathrm{Nx}$ & $\begin{array}{l}\text { Невозможно оценить состояние регионарных } \\
\text { лимфатических узлов }\end{array}$ & $\begin{array}{l}\text { Невозможно оценить состояние регионарных } \\
\text { лимфатических узлов }\end{array}$ \\
\hline No & $\begin{array}{l}\text { Отсутствие метастазов в регионарных } \\
\text { лимфатических узлах }\end{array}$ & $\begin{array}{l}\text { Отсутствие метастазов в регионарных } \\
\text { лимфатических узлах }\end{array}$ \\
\hline N1 & $\begin{array}{l}\text { Метастазы в параректальных лимфатических } \\
\text { узлах }\end{array}$ & Метастазы в регионарных лимфатических узлах \\
\hline$\cdot$ N1a & & $\begin{array}{l}\text { Метастазы в паховых, мезоректальных и/или } \\
\text { внутренних подвздошных лимфоузлах }\end{array}$ \\
\hline$\cdot \mathrm{N} 1 \mathrm{~b}$ & & $\begin{array}{l}\text { Метастазы в наружных подвздошных } \\
\text { лимфоузлах }\end{array}$ \\
\hline$\cdot \mathrm{N} 1 \mathrm{c}$ & & $\begin{array}{l}\text { Метастазы в наружных подвздошных и паховых, } \\
\text { мезоректальных и/или внутренних подвздош- } \\
\text { ных лимфоузлах }\end{array}$ \\
\hline N2 & $\begin{array}{l}\text { Метастазы во внутренних подвздошных и/или } \\
\text { паховых лимфатических узлах с одной стороны }\end{array}$ & - \\
\hline N3 & $\begin{array}{l}\text { Метастазы в параректальных и/или внутренних } \\
\text { подвздошных и/или паховых лимфатических } \\
\text { узлах с двух сторон }\end{array}$ & - \\
\hline \multicolumn{3}{|c|}{ Параметр М-характеристика отдаленных метастазов } \\
\hline Mx & \multicolumn{2}{|c|}{ Наличие отдаленных метастазов установить невозможно } \\
\hline MO & \multicolumn{2}{|l|}{ Отсутствие отдаленных метастазов } \\
\hline M1 & \multicolumn{2}{|l|}{ Наличие отдаленных метастазов } \\
\hline
\end{tabular}

Таблица 2. Стадирование рака анального канала и перианальной кожи по системе TNM

\begin{tabular}{|c|c|c|c|c|c|c|c|}
\hline & & \multicolumn{3}{|c|}{ 7-я редакция TNM } & \multicolumn{3}{|c|}{ 8-я редакция TNM } \\
\hline & Стадия & $\mathrm{T}$ & $\mathbf{N}$ & $M$ & $\mathrm{~T}$ & $\mathbf{N}$ & $M$ \\
\hline Стадия 0 & & Tis & No & Mo & Tis & No & Mo \\
\hline Стадия I & & $\mathrm{T} 1$ & No & MO & $\mathrm{T} 1$ & No & MO \\
\hline Стадия IIA & & $\mathrm{T} 2,3$ & No & Mo & $\mathrm{T} 2$ & No & Mo \\
\hline Стадия IIB & & & & & T3 & No & MO \\
\hline \multirow[t]{6}{*}{ Стадия III } & \multirow[t]{2}{*}{ IIIA } & $\mathrm{T} 1-3$ & N1 & MO & $\mathrm{T} 1$ & N1 & MO \\
\hline & & $\mathrm{T} 4$ & No & MO & $\mathrm{T} 2$ & $\mathrm{~N} 1$ & MO \\
\hline & \multirow[t]{2}{*}{ IIIB } & $\mathrm{T} 1-4$ & N2-3 & Mo & $\mathrm{T} 4$ & NO & Mo \\
\hline & & T4 & $\mathrm{N} 1$ & MO & & & \\
\hline & \multirow[t]{2}{*}{ IIIC } & & & & T3 & N1 & Mo \\
\hline & & & & & $\mathrm{T} 4$ & N1 & MO \\
\hline Стадия IV & & Т любое & N любое & M1 & Т любое & N любое & M1 \\
\hline
\end{tabular}




\section{2. ДИАГНОСТИКА}

Диагноз рака анального канала основывается на данных осмотра, результатах инструментальных методов обследования и патоморфологическом заключении. Первичное обследование должно быть проведено до всех видов лечения и включает в себя:

1) биопсию опухоли с последующим морфологическим исследованием;

2) МРТ малого таза без в/в контрастирования (в/в контрастирование не повышает информативность МРТ малого таза при обследовании по поводу рака анального канала);

3) КТ органов брюшной полости с в/в контрастированием, при недоступности КТ -УЗИ органов брюшной полости;

4) КТ органов грудной клетки, при недоступности КТ - рентгенография органов грудной клетки;

5) сбор анамнеза и физикальный осмотр (включает осмотр кожи перианальной области, пальцевое ректальное исследование, пальпацию паховых лимфатических узлов; гинекологический осмотр);

6) пункцию паховых лимфатических узлов при подозрении на их метастатическое поражение;

7) ректоскопию или аноскопию (ректоскопия бывает технически невыполнима из-за размеров опухоли, выраженного болевого синдрома);

8) осмотр гинеколога, мазок с шейки матки с последующим цитологическим исследованием (у женщин для исключения синхронного поражения шейки матки, которое часто наблюдается при ВПЧ-ассоциированном раке анального канала);

9) онкомаркеры: SCC;

10) клинический анализ крови с подсчетом лейкоцитарной формулы и количества тромбоцитов;

11) биохимический анализ крови с исследованием показателей функции печени, почек;

12) лапароскопию при подозрении на диссеминацию опухоли по брюшине, если её выявление влияет на тактику лечения;

13) МРТ или КТ головного мозга с в/в контрастированием при подозрении на метастатическое поражение головного мозга;

14) ПЭТ/КТ при подозрении на наличие метастазов по данным КТ или МРТ в случаях, когда их подтверждение принципиально меняет тактику лечения;

15) биопсию метастазов под контролем УзИ/КТ при подозрении на их наличие по данным КТ или МРТ в случаях, когда их подтверждение принципиально меняет тактику лечения;

16) ЭКГ;

17) остеосцинтиграфию при подозрении на метастатическое поражение костей скелета;

18) анализ на ВИЧ-инфекцию (при положительном результате - анализ для определения количества CD4 клеток). 


\section{3. ЛЕЧЕНИЕ}

Лечение плоскоклеточного рака анального канала и кожи перианальной области проводится по схожим принципам. Для подавляющего большинства пациентов (за исключением прединвазивных и диссеминированных форм заболевания) основным и единственным методом является ХлТ.

Лечение аденокарциномы анального канала проводится в соответствии с принципами лечения рака нижне-ампулярного отдела прямой кишки и рассматривается в соответствующем разделе рекомендаций.

\section{1. Лечение локализованных форм}

\subsection{1. Хирургическое лечение}

Хирургическое лечение имеет ограниченные показания и может применяться только в отдельных случаях.

1. Хирургическое лечение в объёме местного иссечения возможно только при прединвазивном раке анального канала сTisNOMO и при раке перианальной кожи сTisT1N0M0 умеренной или высокой степени дифференцировки без инвазии сфинктерного аппарата. В отдельных случаях по решению мультидисциплинарной комиссии возможно хирургическое лечение рака перианальной кожи сT2NOMO при высокой или умеренной степени дифференцировки опухоли.

2. Хирургическое лечение в объёме цилиндрической (экстралеваторной) брюшно-промежностной экстирпации прямой кишки может быть выполнено в следующих случаях:

- в случаях массивных жизнеугрожающих кровотечений, связанных с опухолевым процессом, не купирующихся консервативно (подобные осложнения не характерны для рака анального канала и встречаются крайне редко);

- при наличии у пациента в анамнезе лт области малого таза, после консультации лучевого терапевта о невозможности проведения повторного курса ЛТ и пересмотра топометрических карт предшествующей лт (в ряде случаев проведение повторных курсов ЛТ может быть выполнимо);

- при наличии рецидива рака анального канала после ХлТ.

С учетом локализации опухоли у больных плоскоклеточным раком анального канала необходимо выполнение только цилиндрической (экстралеваторной) брюшно-промежностной экстирпации прямой кишки. Выполнение брюшно-промежностной экстирпации прямой кишки без пересечения леваторов у основания не допускается.

3. Хирургическое лечение в объёме сигмостомии перед проведением ХлТ показано пациентам со следующими осложнениями опухолевого процесса:

- кишечная непроходимость на фоне опухолевого стеноза;

- свищи (ректовагинальный, наружный кишечный и т. д.);

- паратуморальные абсцессы (при наличии абсцесса должен быть рассмотрен вопрос о его дренировании до начала ХлТ). 
4. По решению мультидисциплинарной команды возможно хирургическое лечение по поводу олигометастатического заболевания.

\subsection{2. Химиолучевая терапия}

ХЛТ является основным методом лечения больных раком анального канала. При корректном проведении лечения у 80-90\% пациентов можно ожидать полной регрессии опухоли. ХЛТ должна рассматриваться как основной метод лечения у всех больных неметастатическим плоскоклеточным раком анального канала.

Проведение ЛТ без ХТ возможно только при наличии объективных задокументированных противопоказаний к использованию всех химиопрепаратов, включённых в возможные схемы лечения.

У больных метастатическим раком анального канала возможно проведение ХлТ с целью снижения симптомов и уменьшения риска осложнений со стороны первичной опухоли.

ЛТ проводится в РОД 1,8-2,0 Гр, СОД должна составлять от 40-44 Гр на зону регионарного метастазирования; на зону первичной опухоли - в пределах 48-58 Гр, но не менее 48 Гр для T1-Т2 и не менее 54 Гр для Т3-Т4. СОД зависит от стадии заболевания. Перерывы в курсе лТ допустимы только при наличии задокументированных осложнений 3 степени и выше, которые не купируются на фоне консервативного лечения. Прерывание курса Лт без попытки лечения осложнений не допускается. В случае перерыва курс лТ должен быть возобновлён, как только степень проявления осложнений снизится до 2 и ниже. Увеличение времени перерыва может оказать выраженное негативное влияние на результаты лечения. Проведение курсов ЛТ с запланированным перерывом не допускается.

При отсутствии противопоказаний на фоне ЛТ следует проводить ХТ фторпиримидинами и митомицином. Альтернативные схемы допустимо использовать при наличии противопоказаний к использованию митомицина. Схемы ХТ приведены в табл. 3.

Таблица 3. Схемы XT для химиолучевой терапии рака анального канала и перианальной области

\begin{tabular}{|c|c|c|}
\hline Режим & Препарат & Доза и дни введения \\
\hline \multirow[t]{2}{*}{ Нигро } & Митомицин & 10-12 мг/м² (максимально 20 мг) в/в струйно в 1-й день \\
\hline & Фторурацил & $\begin{array}{l}1000 \text { мг/м²/сут. в/в 24-часовая инфузия в 1-й, 2-й, 3-й, } \\
\text { 4-й и 29-й, 30-й, 31-й, 32-й дни }\end{array}$ \\
\hline \multirow[t]{2}{*}{$\begin{array}{l}\text { Митомицин + } \\
\text { капецитабин }\end{array}$} & Митомицин & $\begin{array}{l}10-12 \text { мг/м² (максимально } 20 \text { мг) в/в струйно или капельно } \\
\text { в 1-й день }\end{array}$ \\
\hline & Капецитабин & По 825 мг/м² внутрь ×2 раза в сутки в дни облучения \\
\hline \multirow[t]{2}{*}{$\begin{array}{l}\text { Фторурацил+ } \\
\text { цисплатин }\end{array}$} & Цисплатин & $\begin{array}{l}60 \text { мг/м² в/в капельно на фоне пре- и постгидратации } \\
\text { в 1-й и 29-й дни }\end{array}$ \\
\hline & Фторурацил & $\begin{array}{l}1000 \text { мг/м²/сут. в/в 24-часовая инфузия в 1-й, 2-й, 3-й, } \\
\text { 4-й и 29-й, 30-й, 31-й, 32-й дни }\end{array}$ \\
\hline
\end{tabular}




\section{Принципы лучевой терапии}

- дистанционная ЛТ проводится ежедневно фотонами 6-18 МэВ;

- оптимальным методом является трёхмерная конформная лT (3DCRT), или модулированная по интенсивности ЛТ (IMRT);

- предлучевая топометрическая подготовка включает в себя выполнение КТ и МРТ исследований, давность которых к моменту начала лечения не должна превышать 30 дней; на основании представленных данных формируется план облучения;

- объем облучения включает в себя первичную опухоль и регионарные лимфоузлы; облучение зоны регионарного метастазирования должно включать параректальные, обтураторные, нижние брыжеечные, внутренние и наружные подвздошные, паховые лимфоузлы;

- верхняя граница поля облучения локализуется на уровне L5-S1 позвонков;

- нижняя граница поля облучения - 2,5 см ниже дистального края первичной опухоли;

- на первом этапе проводится ЛТ в РОД 2 Гр до СОД 40-46 Гр на первичную опухоль и зоны регионарного метастазирования;

- на втором этапе проводится локальное облучение, т. е. «буст» на зону исходно определявшихся очагов заболевания. «Буст» проводится в РОД 2 Гр, СОД зависит от исходной распространённости заболевания (СОД 48-52 Гр при Т1-Т 2, СОД 54-60 Гр при Т3-Т4);

- дистанционная ЛТ начинается с объемного 3D планирования и выполняется по технологии конформной лТ (3DCRT) или ее усовершенствованных вариантов: IMRT, объемно-модулированная радиотерапия (VMAT). Точность воспроизведения условий лТ контролируется с помощью системы портальной визуализации в мегавольтном пучке линейного ускорителя электронов ОВI (on board imager) и использования конического киловольтного пучка рентгеновского излучения (СВСТ);

- при технической доступности возможно проведение курса ЛТ с применением протонов/тяжелых ионов энергией облучения 250 МэВ.

По решению мультидисциплинарной команды допустимо использование стереотакстического лучевого воздействия при лечении олигометастатического заболевания.

\subsection{3. Химиолучевая терапия у пациентов с ВИЧ-инфекцией}

Пациентам с ВИЧ-инфекцией лечение проводится без корректировки доз препаратов на фоне адекватной противовирусной терапии при уровне CD4>200. У пациентов C CD4<200 дозы химиопрепаратов и СОД ЛТ должны определяться индивидуально по решению мультидисциплинарной команды. 


\section{2. Лечение диссеминированных форм: плоскоклеточный рак анального канала и перианальной кожи $\mathrm{T}_{\text {любое }} \mathrm{N}_{\text {любое }} \mathrm{M1}$}

\subsection{1. Лекарственное лечение является основным методом лечения диссеминированных и метастатических форм}

\subsubsection{1. Химиотерапия 1 линии}

ХТ является основным методом лечения только у больных диссеминированным раком анального канала. В первой линии ХT рекомендуется использование комбинации карбоплатина и паклитаксела (табл. 4). Лечение проводят до прогрессирования или непереносимой токсичности.

Таблица 4. Схемы лекарственной терапии рака анального канала и перианальной области

\begin{tabular}{|c|c|c|c|c|c|}
\hline Режим & Препарат & Разовая доза & Путь введения & $\begin{array}{l}\text { Дни } \\
\text { введения }\end{array}$ & $\begin{array}{l}\text { Периодич- } \\
\text { ность лечения }\end{array}$ \\
\hline \multicolumn{6}{|l|}{ 1-я линия } \\
\hline \multirow{4}{*}{$\begin{array}{l}\text { Карбоплатин+ } \\
\text { паклитаксел }\end{array}$} & Карбоплатин & AUC 5 & $\mathrm{~B} / \mathrm{B}$ & 1 & \multirow[t]{2}{*}{ Каждые 3 нед. } \\
\hline & Паклитаксел & $175 \mathrm{Mr} / \mathrm{M}^{2}$ & $\mathrm{~B} / \mathrm{B}$ & 1 & \\
\hline & Карбоплатин & AUC 5 & $\mathrm{~B} / \mathrm{B}$ & 1 & \multirow[t]{2}{*}{ Каждые 4 нед. } \\
\hline & Паклитаксел & $80 \mathrm{Mr} / \mathrm{M}^{2}$ & $\mathrm{~B} / \mathrm{B}$ & $1,8,15$ & \\
\hline \multicolumn{6}{|c|}{ 2-я и последующие линии } \\
\hline \multirow{2}{*}{$\begin{array}{l}\text { Фторурацил+ } \\
\text { цисплатин }\end{array}$} & Цисплатин & $60 \mathrm{Mr} / \mathrm{M}^{2}$ & $\mathrm{~B} / \mathrm{B}$ & 2 & \multirow[t]{2}{*}{ Каждые 3 нед. } \\
\hline & Фторурацил & 1000 мг/м²/сут. & $\begin{array}{l}\text { В/в 24-часовая } \\
\text { инфузия }\end{array}$ & $1,2,3,4$ & \\
\hline \multirow[t]{4}{*}{ FOLFCIS } & Цисплатин & $40 \mathrm{Mr} / \mathrm{M}^{2}$ & $\mathrm{~B} / \mathrm{B}$ & 1 & \multirow[t]{4}{*}{ Каждые 2 нед. } \\
\hline & $\begin{array}{l}\text { Кальция } \\
\text { фолинат }\end{array}$ & $400 \mathrm{Mr} / \mathrm{M}^{2}$ & $\mathrm{~B} / \mathrm{B}$ & 1 & \\
\hline & \multirow[t]{2}{*}{ Фторурацил } & $400 \mathrm{Mr} / \mathrm{M}^{2}$ & В/в струйно & 1 & \\
\hline & & $2000 \mathrm{Mr} / \mathrm{M}^{2}$ & $\begin{array}{l}\text { В/в инфузия } \\
46-48 \text { часов }\end{array}$ & 1 & \\
\hline \multirow[t]{3}{*}{$\mathrm{mDCF}$} & Цисплатин & $40 \mathrm{Mr} / \mathrm{M}^{2}$ & $\mathrm{~B} / \mathrm{B}$ & 1 & \multirow[t]{3}{*}{ Каждые 2 нед. } \\
\hline & Доцетаксел & $40 \mathrm{Mr} / \mathrm{M}^{2}$ & $\mathrm{~B} / \mathrm{B}$ & 1 & \\
\hline & Фторурацил & $2000 \mathrm{Mr} / \mathrm{M}^{2}$ & $\begin{array}{l}\text { В/в инфузия } \\
46-48 \text { часов }\end{array}$ & 1 & \\
\hline \multirow{2}{*}{\multicolumn{2}{|c|}{ Пембролизумаб }} & $200 \mathrm{Mr}$ & \multirow{2}{*}{$\begin{array}{l}\text { В/в инфузия } \\
\text { в течение } 30 \text { мин. }\end{array}$} & 1 & Каждые 3 нед. \\
\hline & & $400 \mathrm{Mr}$ & & 1 & Каждые 6 нед. \\
\hline \multirow{2}{*}{\multicolumn{2}{|c|}{ Ниволумаб }} & $240 \mathrm{Mr}$ & \multirow{2}{*}{$\begin{array}{l}\text { В/в инфузия } \\
\text { в течение } 120 \text { мин. }\end{array}$} & 1 & Каждые 2 нед. \\
\hline & & 480 мг & & 1 & Каждые 4 нед. \\
\hline
\end{tabular}




\subsubsection{2. Лекарственная терапия 2-й линии}

Рекомендуется использование комбинации фторурацила и цисплатина (предпочтительно), либо лечение по схеме $\mathrm{MDCF}$ (табл. 4).

При повышенном уровне экспрессии PDL в опухоли (CPS >1) рекомендуется назначение пембролизумаба в одном из режимов: по 200 мг 1 раз в 3 нед. или по 400 мг 1 раз в 6 нед. или ниволумаба 240 мг 1 раз в 2 нед. или 480 мг 1 раз в 4 нед.

\subsubsection{2. Лекарственная терапия 3-й и последующих линий}

После прогрессирования процесса на 2-й линии лечения пациентам в удовлетворительном общем состоянии возможно проведение третьей и последующих линий лечения. Конкретные рекомендации в отношении характера лекарственной терапии 3-й и последующих линий отсутствуют. Выбор терапии осуществляется по усмотрению врача. Применяют препараты, не использованные в терапии 1-й линии, как в монорежиме, так и в комбинациях: производные платины, иринотекан, гемцитабин, таксаны, капецитабин или 5-фторурацил в виде суточных инфузий, из препаратов таргетной терапии - цетуксимаб, панитумумаб (учитывая редкость KRAS мутаций при плоскоклеточном раке анального канала). В 3-й и последующих линиях XТ возможно применение анти-PD - 1 антител: ниволумаба по 240 мг или 3 мг/кг 1 раз в 2 нед. или по 480 мг 1 раз в 4 нед.; при PD-L1 позитивных опухолях (CPS $\geq 1 \%)$ - пембролизумаба по 200 мг 1 раз в 3 нед. или по 400 мг 1 раз в 6 нед. В Российской Федерации применение анти-PD -1 ингибиторов по данному показанию не зарегистрировано.

\section{4. НАБЛЮДЕНИЕ}

\section{1. Оценка эффекта химиолучевой терапии}

Первое контрольное обследование и МРТ малого таза должно быть выполнено через 12 нед. после завершения ХЛТ. Вне зависимости от результатов обследования наблюдение продолжается до 26 нед. после завершения ХЛТ. Решение о необходимости операции должно приниматься не раньше 26 нед. после завершения ХЛТ. Критериями рецидива/продолженного роста рака анального канала являются:

- морфологическая верификация остаточной опухоли через 26 нед. после завершения ХлТ или

- $\quad$ рост остаточной опухоли при проведении двух последовательных МРТ малого таза (например, рост остаточной опухоли по данным МРТ через 26 нед. после завершения ХЛТ по сравнению с данными МРТ через 12 нед. после завершения ХЛТ) или

- сочетание данных 2 и более диагностических методов (МРТ малого таза, ПЭТ/КТ, рост онкомаркера SCC, эндоректального УзИ) +решение мультидисциплинарного консилиума о наличии остаточной опухоли.

При соблюдении всех описанных выше условий и постановке диагноза рецидива/продолженного роста рака анального канала показано хирургическое лечение в объёме цилиндрической (экстралеваторной) брюшно-промежностной экстирпации прямой кишки. 


\section{2. Принятие решения о проведении хирургического лечения в срок до 26 недель}

В следующих клинических ситуациях возможно принятие решения о проведении хирургического лечения в срок до 26 нед. с момента завершения ХЛТ (но не ранее 12 нед. смомента её завершения):

- при наличии полной анальной инконтиненции;

- если лт была ранее проведена со значительными нарушениями клинических рекомендаций и имеются данные 2 и более диагностических методов (МРТ малого таза, ПЭТ/КТ, рост онкомаркера SCС, эндоректального УЗИ) + решение мультидисциплинарного консилиума) о наличии остаточной опухоли;

- при наличии признаков остаточной опухоли через 12 нед. после завершения ХлТ повторное обследование может быть назначено ранее 26 нед., но с интервалом не менее 4 нед. При выявлении отрицательной динамики (рост остаточной опухоли по данным МРТ либо отсутствие регрессии опухоли по данным МРТ в сочетании с морфологической верификацией опухоли) показано проведение хирургического лечения в объёме экстралеваторной экстирпации прямой кишки.

\section{2. Наблюдение за пациентами после радикального лечения}

Обследование:

- пальцевое исследование прямой кишки;

- аноскопия;

- пальпация паховых областей;

- гинекологический осмотр для женщин +цитологический мазок;

- УзИ печени, малого таза, паховых лимфоузлов;

- МРТ малого таза;

- КТ органов грудной клетки (предпочтительно) либо рентгенография органов грудной клетки;

- маркёр SCC;

- КТ органов брюшной полости с в/в контрастированием.

Наблюдение за больным является пожизненным. Обязательное обследование выполняется каждые 3 мес. в течение первых 2 лет после завершения ХлТ, каждые 6 мес.-в течение 3-го года наблюдения и далее - ежегодно. Исключение составляет КТ органов грудной клетки, КТ органов брюшной полости с в/в контрастированием, цитологический мазок шейки матки, которые выполняются ежегодно. Дополнительное обследование назначается по показаниям, в зависимости от результатов основного обследования. 